vertebra and the os occipitis. The whole trunk became flaccid and dead immediately, and the pulsation of the heart was no longer perceived.

Artificial respiration neither restored the beating of the heart, nor occasioned bleeding when the thigh was cut.

Experiment 4.-Destruction of the cervical portion of the spinal marrow as low as the first dorsal vertebra.

The neck immediately became flaccid and dead' the fore extremities insensible, and the heart feeble. Artificial respiration began at three minutes; increased the pulsation of the heart in frequency and strength. The carotids became full and red.

Experiment 5.-Destruction of dorsal portion of spinal marrow, the wire being introduced from the first dorsal to the first lumbar vertebra. The head, neck, and posteriors continued alive. Respiration continued by diaphragm. In five minutes a foot was amputated, but there was no bleeding : at six minutes red bleeding followed amputation of the leg: at fifteen minutes the animal continued to live and breathe, and there was red bleeding.

This is not always the result, for often the destruction of the dorsal portion is followed by all the signs of arrested circulation.

Experiment 6.-Destruction of the lumbar portion: all the hinder parts of the body appeared dead, but the rest continued alive. At eight minutes the foot was amputated, but there was no bleeding : at fifteen minutes neither respiration nor the pulsation of the heart had ceased. The animal supported itself on its fore extremities.

All these experiments were repeated on rabbits ten days old.

Results, 1st.-Circulation was continued after division of the spinal marrow at the occiput.

2nd. Circulation was continued after decapitation, by aid of artificial respiration.

3rd. When the whole spinal marrow was destroyed, the circulation at once ceased, and artificial respiration produced no effect, neither did the thigh cut at three and at seven minutes bleed.

The results are the same when the spinal marrow is cut, without decapitation.

\section{ON THE USE OF THE SPECULUM.}

\section{BY JONATHAN TOOGOOD, ESQ.}

gENIOR SURGEON TO THE BRIDGEWATER INFIRMARY.

During a short visit to Paris in the summer of 1834, I had many opportunities of seeing $M$. Ricord use his speculum, and so satisfied was I of the great advantage of that instrument, that $I$ determined to employ it on all proper occasions.

On my return, $I$ found a patient in the Bridgewater Infirmary, who had been long suffering from a disease of the uterus, which was considered of a cancerous and hopeless nature. The speculum displayed thickening and enlargement of the cervix uteri, with chronic inflammation and incipient ulceration. The diseased parts were slightly rubbed over daily with the nitrate of silver, after which some charpie dipped in a saturnine lotion was applied, and in a month the patient was discharged cured, and has had no return of the disease since.

The next case in which I used it was that of a lady upwards of sixty, who had been suffering for six years with pain in the back and region of the uterus, which increased to such a degree as the day advanced, as to oblige her to give up all exercise, and betake herself to her bed at a very early hour every evening. Occasionally she had some discharge of blood and mucus; her general health was much deranged. She had been long under medical treatment, and employed a great variety of remedies without deriving the least benefit, and had abandoned all hope of cure. An examination per vaginam had been made, but nothing could be learnt by it. I persuaded her to allow me to use the speculum, which discovered an excrescence about the size of a walnut, projecting through the os uteri, which at once explained the feeling of distension so constantly distressing, and all her other symptoms. Without withdrawing the instrument, I carried a pair of forceps, which I have had made for such purposes, through the blades of the speculum, and removed the tumor without difficulty. The part from which this vascular excrescence grew was touched daily with lunar caustic three or four times, when the patient was completely cured. It is now more than a year since this operation was performed, and the patient has continued in the full enjoyment of health.

Another case has lately fallen under my care, of a young and delicate lady, who was attacked with pain and tenderness in the region of the uterus, attended with considerable discharge, occasional sickness, and general constitutional disturbance; in fact, all the symptoms described by Dr. Gooch in his paper on "Irritable Uterus." She had consulted many eminent practitioners, and had submitted with exemplary patience to every plan of treatment which had been recommended, without deriving the least benefit. At last she placed herself under the care of a celebrated physician in London, whose attention had been exclusively devoted to female diseases, and although she strictly followed the plan laid down, and had the advantage of his regular attendance for six months, her complaint remained exactly the same at the end of that period. Under these circumstances she submitted to the employment of the speculum, which showed that her long-continued suffering arose from inflammation and enlargement of the cervix uteri and ulceration of its surface. The diseased parts were slightly rubbed over with lunar caustic, which was repeated every fourth day, and intermediately an injection of sulphate of alum was employed. These remedies, with attention to the general health, completely restored her in two months.

The advantages of this instrument are too obvious to insist on; it enables the practitioner to obtain a correct view of the disease of which he could only have an imperfect idea without its use, whilst at the same time it affords peculiar facility for performing operations and applying dressings. It has been said that patients will not submit to its employment, but I have never yet met with a case in which it was necessary to use it, in which the most delicate woman objected to its use. I have used it also in diseases of the rectum advantageously. 